liver function tests. Patients with central line placement and heparin exposure were associated strongly with mild thrombocytopenia $(p<0.0001)$. Drug therapies that were correlated with thrombocytopenia included Heparin, Protonix, Lasix, Ativan and Zofran and antibiotics such as Vancomycin, Cephalosporins and Levaquin.

Conclusion Drug regimens should be evaluated daily for minimization of adverse drug events including thrombocytopenia. Once the diagnosis is suspected, clinicians should identify the medication and/or risk factors causing secondary thrombocytopenia to assess the timeline of development. Co morbidity associated with thrombocytopenia was sepsis syndrome, liver disorder, alcoholism and atrial fibrillation. Medications commonly associated with druginduced thrombocytopenia include glycoprotein IIb/IIIa inhibitors, cinchona alkaloids, antibiotics, anticonvulsants, and heparin. Thrombocytopenia generally resolved in most patients with critical management of the disease and discontinuation of the offending medication.

\section{S75 HIGH DOSE VITAMIN D SUPPLEMENTATION IMPROVES EXTRAVASCULAR LUNG WATER INDEX AND IN-VIVO TREG AND LL37 RESPONSES POST-0ESOPHAGECTOMY}

doi:10.1136/thoraxjnl-2012-202678.081

'RCA Dancer, 'D Parekh, ${ }^{2} \mathrm{AR}$ Martineau, ${ }^{3} \mathrm{GD}$ Perkins, 'DR Thickett. 'University of Birmingham, Birmingham, UK; ${ }^{2}$ Bartsthe London School of Medicine and Dentistry, London, UK; ${ }^{3}$ Warwick Medical School, Coventry, UK

Acute Lung Injury occurs in around $25 \%$ of patients post 2 stage oesophagectomy. We have previously shown that levels of $25-\mathrm{OH}$ vitamin $\mathrm{D}$ are low in these patients and that those patients with the lowest levels of vitamin D are more likely to develop Acute Lung Injury post-operatively. In vitro, vitamin $\mathrm{D}$ has been shown to promote the differentiation of regulatory $\mathrm{T}$ cells (Treg) and the expression of LL37 (cathelicidin), an antimicrobial peptide.

We have given 13 patients a one-off high dose vitamin $\mathrm{D}$ supplement 3-14 days prior to oesophagectomy. Numbers of circulating regulatory $\mathrm{T}$ cells were analysed both pre-and post-supplementation. Plasma levels of LL37 were measured by ELISA. Extravascular Lung Water Index (EVLWi) was measured pre-operatively, post-operatively and on the day following surgery. Changes in LL37 and EVLWi were compared with results from a cohort of patients $(n=50)$ who had not been supplemented.

Pre-operative vitamin D levels between the two groups were significantly different. The proportion of CD3 $+\mathrm{CD} 4+\mathrm{T}$ cells which were CD25+CD127loFoxP3+ increased post vitamin D supplementation (median pre vitamin $\mathrm{D}=5.8$, median post vitamin $\mathrm{D}=7.3, \mathrm{p}=0.028$ ). Whilst levels of LL37 decreased post-operatively in the cohort who had not received vitamin $\mathrm{D}$, levels were maintained in supplemented patients. Post-operative EVLWi measured lower than pre-operative values in patients receiving vitamin $\mathrm{D}$ supplementation. This contrasts with patients who did not receive vitamin $\mathrm{D}$ supplementation in whom an increase in EVLWi was seen. This difference persists on post-operative day 1, with a net increase being seen in patients who did not receive vitamin $\mathrm{D}$ and a net decrease in those who did (see table).

Conclusions A stat high dose vitamin D supplement restored vitamin D levels in the week before oesophagectomy. This was associated with elevated circulating Treg levels in vivo and an increase in post-operative plasma LL37 expression suggesting the vitamin D supplementation was biologically active.

Patients receiving vitamin $\mathrm{D}$ had no perioperative increase in extravascular lung water compared to our historical cohort suggesting that vitamin $\mathrm{D}$ protects against alveolar epithelial damage perhaps in part due to effects on circulating Treg cells and anti-microbial peptide production.
Abstract S75 Table 1 Effects of Vitamin D supplementation on median values of Vitamin D, EVLWi and LL37

\begin{tabular}{lccc}
\hline & $\begin{array}{l}\text { Vitamin D } \\
\text { supplementation }\end{array}$ & $\begin{array}{l}\text { No Vitamin D } \\
\text { supplementation }\end{array}$ & $\begin{array}{l}\text { p value } \\
\text { (Mann-Whitney U test) }\end{array}$ \\
\hline 25-OH Vitamin D level & $76 \mathrm{nmol} / \mathrm{L}$ & $25 \mathrm{nmol} / \mathrm{L}$ & 0.001 \\
Change in EVLWi D0 post-op & -1.0 & 2.0 & 0.004 \\
Change in EVLWi D1 post-op & -1.0 & 1.0 & 0.023 \\
\% change in plasma LL37 as & 10.9 & -17.8 & 0.003 \\
proportion of total protein & & & \\
post-op & & & \\
\hline
\end{tabular}

\section{S76 PROTEOLYTIC CLEAVAGE OF ELAFIN BY 20S PROTEASOME MAY CONTRIBUTE TO INFLAMMATION IN ACUTE LUNG INJURY}

doi:10.1136/thoraxjnl-2012-202678.082

${ }^{1}$ A Kerrin, 'S Weldon, ${ }^{2} \mathrm{~A}$ Chang, 'T Craig, ${ }^{3} \mathrm{AJ}$ Simpson, 'C O'Kane, 'DF McAuley, ${ }^{1} \mathrm{C}$ Taggart. Queen's. 'University Belfast, Belfast, United Kingdom; 'National Institutes of Health, Bethesda, USA; ${ }^{3}$ University of Newcastle, Newcastle, united Kingdom

The Aim of this study was to characterise temporal changes in elafin concentration in patients with acute lung injury (ALI) and to evaluate whether a decrease in elafin levels is due to elevated protease activity. Previous work has shown that unregulated protease activity can cause proteolytic cleavage of elafin, impairing the innate immune function of the protein. Bronchoalveolar lavage fluid (BALF) was obtained from patients with ALI within 48 hours of onset of ALI (day 0), at day 3 and at day 7. Elafin levels were quantified by ELISA Elafin susceptibility to proteolytic cleavage by ALI BALF was assessed by Western blot and by HPLC-Mass Spectrometry. Elafin levels were found to be significantly increased at the onset of ALI compared to healthy volunteers and fell significantly by day 7 compared to day 0 . In contrast, levels of secretory leukocyte protease inhibitor (SLPI) did not decrease over time. This decrease in elafin was due to cleavage by the $20 \mathrm{~S}$ proteasome which was significantly increased in ALI BALF. Incubation of ALI BALF with the proteasome inhibitor epoxomicin confirmed that $20 \mathrm{~S}$ proteasome protease activity was responsible for proteolytic cleavage of elafin resulting in diminished anti-elastase activity. In addition, free neutrophil elastase (NE) activity significantly increased in ALI BALF from day 0 to day 7. In conclusion, elafin concentrations decrease within the pulmonary compartment over the course of ALI as a result of proteolytic degradation. This loss of elafin may predispose, in part, to excessive inflammation in ALI.

\section{S77 PRONE POSITIONING AND INTRAVENOUS STEROIDS FOR THE MANAGEMENT OF SEVERE ARDS}

doi:10.1136/thoraxjnl-2012-202678.083

L Gupta, Ben SiuJames. Regional Medical Center of San Jose, San Jose, USA

Introduction Prone positioning and intravenous steroids represents a treatment option in patients with severe ARDS because most of the clinical manifestations in ARDS is secondary to excess fluid in the interstitium and alveoli and presence of inflammatory cells in the fluid.

Objective To assess the impact of prone positioning and steroids in severe ARDS and to assess and identify prognostic factors and potential predictors of mortality in ARDS patients.

Methods This is a retrospective study of 46 patients with severe ARDS from 2009-2011. The patient charts were reviewed and the impact of prone positioning and steroids was observed on severe ARDS patients. The primary outcome measured were ICU length of stay, number of ventilator days and 30 day mortality. 
Various prognostic factors such as history of diabetes, alcoholism, and presence of hypo-albuminemia, coagulopathy, sepsis and lactic acidosis were taken into account. Calculations were made in regards to the Lung Injury Predictive Score (LIPS) as determined by Chest Roentgenogram score, Hypoxemia Score, PEEP Score and Compliance score. The LIPS score was calculated for projecting the severity of ARDS. The use of statins on various outcome measures was also observed.

Results A total of 46 patients were treated in the ICU for ARDS. The number of ventilator days averaged from 5-35 days. The incidence of predisposing conditions as well as risk modifiers was correlated with the LIPS score. All of these patients were on ARDS net protocol and received intravenous antibiotics. Statistical analysis revealed a favourable impact of prone positioning with steroids on the mortality, duration of ICU stay and ventilator days. A significant difference in the LIPS score was noted in patients receiving IV steroids and prone positioning on day 2. Use of statins also influenced the duration of ICU stay and a significant impact on the mortality of this cohort of patients.

Conclusions Prone positioning is an effective adjunct intervention in conjunction with intravenous steroids for treating severe ARDS. It is a valid option for patients with refractory ARDS to conventional treatment. More studies need to be done to validate the impact of statins on different outcome measures in the ICU.

\section{Airway inflammation and infections}

\section{S78 PHENOTYPES OF INDUCED SPUTUM IN DIFFICULT TO TREAT ASTHMA}

doi:10.1136/thoraxjnl-2012-202678.084

R Herzallah. University of Leicester, Leicester, UK

Introduction and Objectives The clinical value of repeated induced sputum tests to monitor airway inflammation in children with severe asthma is contentious. In adult patients with severe asthma a management based on sputum eosinophil counts results in a reduction of exacerbations and fewer hospital admissions (Green R et al, Lancet 2002). This has not been shown in children. Several inflammatory phenotypes are described but clinical management strategies based on these have largely been unsuccessful. It has been suggested that the inflammatory phenotypes in the sputum are unstable over time (Fleming et al, Thorax 2012). We reviewed the results of induced sputum tests done as part of our severe asthma clinic to determine stability of inflammatory phenotypes in our patients.

Methods We reviewed all sputum induction results for children with a diagnosis of asthma who underwent inductions between April 2008 and June 2012 at our centre. Samples were processes using our standard protocol (Pin et al, Thorax 1992).

Samples were classified as eosinophilic ( $>2.5 \%$ eosinophils and $<2.5 \%$ neutrophils), neutrophilic ( $>54 \%$ neutrophils and $<2.5 \%$ Eos); mixed granulocytic ( $>2.5 \%$ eosinophils, $>54 \%$ neutrophils); or paucigranulocytic ( $<2.5 \%$ eosinophils, $<54 \%$ neutrophils) as previously suggested.

Results 34 patients, 19 males and 15 females, with a BTS of 3 to 5 had a total of 86 inductions. 22 patients had 2 inductions and the rest had between 3 and 5 inductions.

18 of the 34 patients had a eosinophilic profile on their first induction and 24 of the 34 patients had a eosinophilic profile in at least one sample. 29 of the 34 patients had $>2.5 \%$ eosinophils (eosinophilc or mixed profile) in at least one sample. Of the remaining five who never had $>2.5 \%$ eosinophils, four had a neutrophic profile and the remaining child had a persistent paucigranulocytic profile.

Using the above classification 25 out of the 34 patients switched phenotype at least once in the duration of the study.
Conclusions There is no treatment based on the neutrophilic phenotype classification therefore the presence and percentage of eosinophils present appears to be the most useful information gained from sputum induction. Based on our data and that of others, classification into several inflammatory phenotypes does not appear clinically useful.

\section{S79 SPUTUM INFLAMMATORY CELL PROFILE IN CHILDREN WITH ACUTE ASTHMA AND FOLLOWING RECOVERY}

doi:10.1136/thoraxjnl-2012-202678.085

KG Staley, G Fisher, CE Brightling, P Bradding, D Parker, EA Gaillard. University of Leicester, Leicester, UK

Background Airway inflammation in children experiencing an acute asthma exacerbation has been little studied. A previous study (1) reported high numbers of sputum inflammatory cells in children with acute asthma and a significantly lower number two weeks after the acute episode. Our principal objective was to study children after a longer follow-up period when the effects of oral steroid therapy has subsided and to compare children with asthma to a healthy control group. Methods We collected sputum from children attending hospital with acute asthma following salbutamol bronchodilation, and, if necessary (and $\mathrm{FEV}_{1}>50 \%$ predicted), after nebulisation with $0.9 \%$ saline. Following recovery, sputum was obtained using hypertonicsaline induction. We also studied a control group of healthy children. Sputum samples were processed within 6 hours and a differential cell-count was obtained by counting inflammatory cells on a cytospin slide (2).

Results Paired sputum samples were obtained from fifteen children (median-age 11 years) and eight controls (median-age 13.5 years). Median number of days between sputum samples for children with asthma was 82 days (range 21-313). Sputum from children with acute asthma had a higher number of inflammatory cells and a higher percentage eosinophil count than control children $(p=0.025,0.015$ respectively). Sputum from children with asthma at recovery had a higher percentage eosinophil count than control children $(p=0.021)$, but total number of inflammatory cells were not significantly different. We did not find a significant decrease in total number of cells nor percentage neutrophils or eosinophils in the sputum of children with asthma between acute episode and recovery [see table 1]. In children with raised sputum eosinophils $(>2.5 \%)$ during acute asthma sputum eosinophilia persisted at recovery.

Conclusion Sputum inflammatory cell counts and percentage eosinophils are elevated in children with acute asthma compared to children without asthma. We found no significant difference in either total or percentage neutrophil or eosinophil count between acute asthma and recovery. In children with eosinophilia $>2.5 \%$ during acute asthma this persisted at recovery. These findings have implications for our understanding of the causes of asthma exacerbations in children.

1. Norzila MZ et al. AJRCCMed 2000:769-774

2. Pin I et al. Thorax 1992:25-29

Abstract S79 Table 1 Table to Illustrate Inflammatory cell Profile of sputum from Children with Asthma during acute exacerbation and at recovery and in Control Children

\begin{tabular}{llll}
\hline Inflammatory cells & $\begin{array}{c}\text { Asthma acute } \\
\text { exacerbation }\end{array}$ & Asthma recovery & \multicolumn{1}{c}{ Control } \\
\hline Total number cells $\left[\times 10^{3} / \mathrm{ul}\right]^{*}$ & $1.35(0.14-13.99)$ & $0.61(0.04-3.22)$ & $0.14(0.06-3.77)$ \\
$\%$ neutrophils* & $77.2(24.5-97.3)$ & $70.3(11-94)$ & $53.6(13.5-96.8)$ \\
$\%$ eosinophils* & $2.8(0.0-54.5)$ & $4.5(0.0-46.0)$ & $0.12(0.0-2.0)$ \\
\hline
\end{tabular}

"values are median with range in parentheses. 